\title{
ANÁLISE EPIDEMIOLÓGICA DE HIPERTENSÃO ARTERIAL SISTÊMICA NA COMUNIDADE QUILOMBOLA DO CURIAÚ NO ESTADO DO AMAPÁ
}

ARTIGO ORIGINAL 
SANTOS FILHO, João Gilberto Sakai 1, PENA, Eduarda Emanuelle da Silva 2, ESPINDOLA, Gabriel de Oliveira ${ }^{3}$, BRITO, Maiara de Fátima de Brito ${ }^{4}$, RESQUE, Rafael Lima ${ }^{5}$, BARBOSA, Robson Carvalho ${ }^{6}$, VANZELER, Tainá Lobato ${ }^{7}$

1 Graduando em Ciências Farmacêuticas pela Universidade Federal do Amapá. ORCID: https://orcid.org/0000-0002-3181-6630

${ }^{2}$ Especialização em Especialização em Perícia Criminal.Instituto de Pós- Graduação e Pesquisa em Ciências da Saúde do Amapá, IECSA, Brasil. Graduação em Ciências Biológicas, Universidade Federal do Amapá, UNIFAP, Brasil. ORCID: https://orcid.org/ 0000-0002-8181-8773

${ }^{3}$ Mestrado em CIÊNCIAS DA SAÚDE, Universidade Federal do Amapá, UNIFAP, Brasil. Especialização em andamento em Biologia Molecular e Genética Forense, Instituto Nacional de Perícias e Ciências Forenses, INFOR, Brasil. Graduação em Ciências Biológicas, Universidade Federal do Amapá, UNIFAP, Brasil. ORCID: https://orcid.org/0000-0002-0749-4627

4 Graduação em Ciências Farmacêuticas pela Universidade Federal do Amapá. ORCID: https://orcid.org/0000-0002-6302-8647

${ }^{5}$ Doutorado em Genética e Biologia Molecular pela Universidade Federal do Pará, UFPA, Brasil. Mestrado em Genética e Biologia Molecular pela Universidade Federal do Pará, UFPA, Brasil. Especialização em Genética e Biologia Molecular pela Universidade Luterana do Brasil, ULBRA, Brasil.Graduação em Farmácia pelo Centro Universitário do Estado do Pará, CESUPA, Brasil. ORCID: https://orcid.org/0000-0002-5769-5734

${ }^{6}$ Mestrado em andamento em Ciências Farmacêuticas, Universidade Federal do Amapá, UNIFAP, Brasil. Especialização em andamento em Curso de Especialização em Perícia Criminal. Instituto de Pós Graduação e Pesquisa em Ciência da Saúde do Amapá, IECSA, Brasil. Graduação em BIOMEDICINA. Faculdade Estácio de Macapá, Estácio Macapá, Brasil. ORCID: https://orcid.org/0000-0001-6826-8327

${ }^{7}$ Mestrado em CIÊNCIAS DA SAÚDE, Universidade Federal do Amapá, UNIFAP, Brasil. Graduação em Ciências Biológicas, Universidade Federal do Amapá, UNIFAP, Brasil. ORCID: https://orcid.org/00000002-0940-0102

RC: 106017

Disponível em: https://www.nucleodoconhecimento.com.br/saude/quilombola-do-curiau 
SANTOS FILHO, João Gilberto Sakai. Et al. Análise epidemiológica de hipertensão arterial sistêmica na comunidade Quilombola do Curiaú no estado do Amapá. Revista Científica Multidisciplinar Núcleo do Conhecimento. Ano. 07, Ed. 01, Vol. 06, pp. 140-153. Janeiro de 2022. ISSN: 2448-0959, Link de acesso: https://www.nucleodoconhecimento.com.br/saude/quilombola-do-curiau

\section{RESUMO}

Com elevada prevalência e baixas taxas de controle a Hipertensão Arterial Sistêmica (HAS) é um dos principais fatores de risco modificáveis para doenças cardiovasculares e um dos mais importantes problemas de saúde pública. Questão norteadora: Qual a prevalência e os fatores associados de HAS na Comunidade Quilombola do Curiaú? Objetivo: O objetivo deste estudo foi a análise epidemiológica da prevalência de HAS e fatores associados a ela na Comunidade Quilombola do Curiaú. Material e Métodos: Trata-se de um estudo descritivo transversal, sendo realizado com 71 voluntários, com idade $\geq 18$ anos e residentes na comunidade. Resultados: A média de idade dos voluntários foi de 49,01 anos, sendo $64,7 \%(n=46)$ do sexo feminino e 35,2 \% ( $n=25)$ do sexo masculino. Observou-se que 32,8\% ( $n=24)$ dos participantes eram hipertensos, sendo $33,4 \%(n=8)$ homens e $66,6 \%(n=16)$ mulheres, entre estes $50 \%(n=12)$ estavam sob terapia com anti-hipertensivos. Dentre os participantes, constatou-se que 70,8\% tinham histórico familiar de hipertensão, além disso, 33,8\% dos voluntários apresentaram níveis pressóricos elevados. Conclusão: Foi observada elevada prevalência de HAS e níveis pressóricos elevados na comunidade quilombola do Curiaú, em destaque para os indivíduos do sexo feminino, pois foram os que mais apresentaram a doença. Também, foi detectado maior prevalência de HAS em pessoas com idade $\geq 60$ anos. Desta forma, é necessário que haja maior atenção a política de saúde pública e a implementação de programas que foquem no tratamento medicamentoso deste quilombo.

Palavras-Chave: Afrodescendentes, Quilombolas, Hipertensão Arterial. 


\section{INTRODUÇÃO}

A HAS é uma doença crônica não transmissível (DCNT), definida por elevação sustentada dos níveis pressóricos ligada a multifatores de agravo, tais como: má alimentação, falta de exercício físico, tabagismo, quantidade excessiva de álcool ou sódio, estresse, obesidade e genética (BARROSO, 2021).

Com elevada prevalência e baixas taxas de controle, a HAS é um dos principais fatores de risco modificáveis para doenças cardiovasculares e um dos mais importantes problemas de saúde pública atualmente (BRANDÃO, 2010). A HAS tende a ser assintomática, causando implicações na dificuldade de diagnóstico precoce e na baixa adesão por parte do paciente ao tratamento prescrito, visto que há medicamentos que apresentam efeitos colaterais, por conseguinte, o controle da HAS torna-se ineficaz (MONTEIRO et al., 2020).

Lobo et al. (2017) constatou dados sobre a população brasileira que apontaram o aumento da prevalência de HAS, onde se destacam a associação com os baixos níveis de escolaridade, mulheres de baixa renda e, nos homens, aqueles que possuíam a renda financeira mais elevada.

No Brasil, a HAS se apresenta com maior prevalência e gravidade em populações com pronunciada descendência africana. Desta forma, a população remanescente de quilombo residente no Curiaú, no Estado do Amapá, pode ser eleita como um ponto de maior atenção neste sentido, visto que apresenta historicamente maior contribuição genética afrodescendente que as populações brasileiras miscigenadas residentes em centros urbanos (LINDHORST et al., 2007; KURIAN; CARDARELLI, 2007; BARROS et al., 2006).

Desta forma, a relevância de um estudo étnico racial relativo às doenças e determinantes sociais é pertinente em populações mais suscetíveis. Neste contexto, a questão norteadora do presente artigo foi: Qual a prevalência e os fatores associados de HAS na Comunidade Quilombola do Curiaú? Logo, o objetivo deste

RC: 106017

Disponível em: https://www.nucleodoconhecimento.com.br/saude/quilombola-do-curiau 
trabalho foi de realizar a análise epidemiológica da HAS e identificar fatores associados à essa doença em moradores da comunidade do quilombo do Curiaú.

Existe uma quantidade singela de estudos sobre a população negra no Brasil, principalmente sobre os afrodescendentes quilombolas, neste sentido o presente estudo contribui para a ampliação da visibilidade das desigualdades que atingem a população quilombola, além de identificar necessidades de saúde e de promoção de intervenções terapêuticas, para assim, ajudar com o planejamento de ações voltadas, não só para prevenção da hipertensão, mas também para o controle da HAS.

\section{MATERIAIS E MÉTODOS}

Tratou-se de um estudo transversal descritivo para análise epidemiológica da HAS na Comunidade Quilombola do Curiaú no Estado do Amapá. Os critérios de inclusão foram: serem adultos (idade igual ou superior a 18 anos), residentes na comunidade do Curiaú que aceitaram participar da pesquisa e assinaram o Termo de Consentimento Livre e Esclarecido (TCLE); e os de exclusão: foram as pessoas não residentes da comunidade, bem como os menores de idade ou os que foram considerados incapazes de decidir pela sua participação. A partir de tais critérios, a amostra consistiu em 71 voluntários residentes na comunidade do Curiaú.

\subsection{COLETA DE DADOS E VARIÁVEIS}

As coletas de dados foram realizadas no período de agosto a dezembro de 2019. As variáveis estudadas foram: sexo (feminino ou masculino); idade e faixa etária (18 a 29 anos, 30 a 59 anos e acima de 60 anos); etnia (europeu, africano, indígena e asiático), situação conjugal, estilo de vida, como: o consumo de álcool e cigarro (esporadicamente, semanalmente, diariamente, não faz uso); doenças presentes: hipertensão arterial sistêmica (HAS) e a existência de outras doenças foram avaliadas por meio de perguntas dicotomizadas (sim ou não); medidas como aferição de pressão arterial e teste de glicemia, além de uso de medicamentos anti-hipertensivos. 
As aferições e a classificação dos níveis da pressão arterial (PA) seguiram o protocolo contido na VII Diretriz Brasileira de Hipertensão Arterial. Foi orientado para que os indivíduos estivessem de bexiga vazia, não praticassem exercícios físicos há pelo menos 60 minutos, não houvessem feito ingestão de bebidas alcoólicas, cigarro, café ou alimentos nos 30 minutos anteriores.

As medidas de pressão arterial foram realizadas duas vezes (com intervalo de 2 minutos entre cada aferição) sendo utilizado o esfigmomanômetro digital da marca OMRON-HEM 6124, segundo os critérios estabelecidos pelas Diretrizes Brasileiras de Hipertensão Arterial (BARROSO et al., 2020). Após repouso entre três e cinco minutos, os participantes ficaram sentados, com as pernas descruzadas, os pés apoiados no chão, o dorso recostado na cadeira e relaxado, com o braço esquerdo apoiado sobre a mesa à altura do coração. Foi considerado como valor pressórico final o resultado da média das duas últimas medidas. Considerou-se dentro dos padrões de normalidade aquelas pessoas com PA cujos níveis tensionais marcassem menos que 140 por $90 \mathrm{mmHg}$.

\subsection{ANÁLISE ESTATÍSTICA}

O programa Biostat 5.0 foi utilizado para a análise estatística dos dados epidemiológicos (média de idade e coeficiente de variação). As variáveis dependentes foram submetidas a tabulação em planilha e analisadas por meio de correlação com o teste de Qui-quadrado (X2) pelo programa Microsoft@ Office Excel 2013.

\subsection{ASPECTOS ÉTICOS}

O projeto de pesquisa foi submetido, de acordo com a Resolução no 466/12 para avaliação na Plataforma Brasil e ao Comitê de Ética em Pesquisa (CEP), localizado na Universidade Federal do Amapá - UNIFAP. A aprovação do estudo está registrada sob o número de identificação CAAE 14952919.5.0000.0003.

RC: 106017

Disponível em: https://www.nucleodoconhecimento.com.br/saude/quilombola-do-curiau 
Os participantes foram esclarecidos sobre o objetivo da pesquisa e orientados para a assinatura do Termo de Consentimento Livre e Esclarecido, autorizando a utilização da sua amostra no estudo.

\section{RESULTADOS E DISCUSSÃO}

O estudo contou com a participação de 73 indivíduos quilombolas residentes na comunidade do Curiaú. Dentre os 73 voluntários que participaram da pesquisa, 71 $(96,2 \%)$ foram os que participaram de todo o processo do estudo. Houve uma redução de $3,7 \%$ de participantes pelo fato de não estarem incluídos nos critérios estabelecidos. A maioria dos participantes era do sexo feminino $46(64,7 \%)$ e 25 $(35,2 \%)$ do sexo masculino. Em relação à idade, os que possuíam a idade de 18 a 29 anos totalizaram $11(15,49 \%)$, de 30 a 59 anos 38 (53,52\%) e maiores de 60 anos totalizaram $22(30,98 \%)$.

Além disso, 85,9\% dos entrevistados declararam ser de origem africana, 9,8\% de origem indígena, 1,4\% se referiram ser de origem europeia e 2,8\% não sabem ou não quiseram informar. No que diz respeito à situação conjugal, observou-se que 42,2 \% são casados e/ou vivem com alguém da mesma comunidade.

Perfil semelhante também foi encontrado no estudo de Santos et al. (2020), realizado com adultos quilombolas na Bahia, onde a variação de idade encontrada entre os participantes foi de 18 a 92 anos, com mediana de 45 anos. A amostra foi prevalentemente composta por mulheres, totalizando $61,1 \%$, destes. Dentre os participantes, $86,5 \%$ se autorreferiram negros e $76,7 \%$ dos indivíduos relataram possuir cônjuge.

Em relação ao perfil dos portadores de HAS, foi possível identificar maior prevalência da doença nas mulheres, cerca de 34,7\% (Tabela 1), além disso, houve uma prevalência significativa na faixa etária de 30-59 anos e de 60 anos ou mais. De acordo com dados da análise da Pesquisa Nacional de Saúde de 2013, mencionados no estudo de Andrade et al. (2015), foi encontrada a prevalência de 21,4\%, sendo maior

RC: 106017

Disponível em: https://www.nucleodoconhecimento.com.br/saude/quilombola-do-curiau 
entre mulheres $24,2 \%$, e em indivíduos com mais de 75 anos de idade $55,0 \%$ e de raça/cor preta $24,2 \%$.

Quadro 1 - Relação HAS - Sexo

\begin{tabular}{|c|c|c|c|}
\hline \multicolumn{2}{|l|}{ VARIÁVEL } & HOMENS & MULHERES \\
\hline \multirow[t]{2}{*}{ Hipertensos } & Sim & $8(32 \%)$ & $16(34,7 \%)$ \\
\hline & Não & 17 & 30 \\
\hline PA elevada & Sim & 8 & 3 \\
\hline Sem diagnóstico de HAS & Não & 9 & 27 \\
\hline \multirow[t]{2}{*}{ PA controlada } & Sim & 2 & 2 \\
\hline & Não & 1 & 5 \\
\hline \multirow{2}{*}{$\begin{array}{l}\text { Histórico de portadores } \\
\text { de HAS }\end{array}$} & Sim & 4 & 13 \\
\hline & Não & 5 & 2 \\
\hline \multirow[t]{2}{*}{ Histórico de HAS } & Sim & 19 & 30 \\
\hline & Não & 6 & 16 \\
\hline
\end{tabular}

Fonte: Dados do autor, 2019

Em um estudo realizado com um nicho de adultos no sertão de Pernambuco, foi evidenciada a prevalência de HAS no quantitativo de $27,4 \%$, se mostrando crescente com o aumento da idade e diminuição do nível de escolaridade, além disso, há maior prevalência da doença nas pessoas de menores classes econômicas (SANTIAGO et al., 2019).

Sobretudo, dentre os que apresentaram HAS, constatou-se que $70,8 \%$ tinham histórico familiar de hipertensão, sendo que as mulheres possuíam maior número de pessoas com HAS em suas famílias, cerca de $76 \%(n=35)$ e, os homens, apresentaram $56 \%$ ( $n=14)$. Não foi observada significativa associação entre histórico familiar de HAS e sexo na comunidade do Curiaú $(p=0,080)$. Em uma pesquisa feita com hipertensos e diabetes associados, cadastrados em uma Estratégia Saúde da 
Família (ESF) de Minas Gerais, identificou-se que 70\% dos entrevistados possuíam histórico familiar de HAS ou DM (PRATES et al., 2020).

Dos participantes no presente estudo diagnosticados como hipertensos $(n=24), 12$ $(50,0 \%)$ estavam em tratamento com anti-hipertensivo da classe ARA (antagonistas dos receptores de angiotensina II) associado a um diurético da classe dos tiazídicos e $60,0 \%(n=6)$ não apresentaram PA controlada, mesmo fazendo uso de medicamentos, sendo $17 \%(n=1)$ do sexo masculino e $83 \%(n=5)$ do sexo feminino. Menezes; Portes e Silva (2020) identificaram, entre os indivíduos hipertensos em seu estudo, que dentre os $73 \%$ que sabiam serem portadores da doença, $95 \%$ destes faziam tratamento, porém apenas $49 \%$ deles mantinham controle pressórico adequado, refletindo a dicotomia do tratamento negligenciado.

No quadro 2, é possível visualizar os resultados de controle dos níveis pressóricos com a aplicação de terapia medicamentosa e, apesar das mulheres possuírem maior autocuidado, principalmente no que diz respeito a procura mais rápido por atendimento, os dados estatísticos não tiveram significância estatística $(p=0,144)$ e mostram que o sexo feminino neste estudo é o que menos possui controle da HAS.

Quadro 2 - Prevalência e controle de HAS, segundo o sexo, na população quilombola do Curiaú.

\begin{tabular}{|c|c|c|c|c|}
\hline \multicolumn{2}{|l|}{ VARIÁVEL } & HOMENS & MULHERES & P- VALOR \\
\hline \multirow[t]{2}{*}{ Hipertensos } & Sim & $8(33,3 \%)$ & $16(66,6)$ & \multirow[t]{2}{*}{0,813} \\
\hline & Não & $17(36,2 \%)$ & $30(63,8 \%)$ & \\
\hline \multirow[t]{2}{*}{ PA elevada } & Sim & $13(52 \%)$ & $10(21,7 \%)$ & \multirow[t]{2}{*}{$0,009^{*}$} \\
\hline & Não & $12(48 \%)$ & $36(78,2 \%)$ & \\
\hline \multirow{2}{*}{$\begin{array}{l}\text { PA elevada } \\
\text { (sem } \\
\text { diagnóstico de } \\
\text { HAS) }\end{array}$} & Sim & $2(25 \%)$ & $6(75 \%)$ & \multirow[t]{2}{*}{0,745} \\
\hline & Não & $12(30,7 \%)$ & $27(69,2 \%)$ & \\
\hline
\end{tabular}

RC: 106017

Disponível em: https://www.nucleodoconhecimento.com.br/saude/quilombola-do-curiau 


\begin{tabular}{|l|l|l|l|l|}
\hline PA controlada & Sim & $2(18,1 \%)$ & $1(9 \%)$ & 0,144 \\
\cline { 2 - 4 } & Não & $1(9 \%)$ & $5(45,4 \%)$ & \\
\hline \multirow{2}{*}{$\begin{array}{l}\text { Histórico de } \\
\text { HAS }\end{array}$} & Sim & $14(56 \%)$ & $35(76 \%)$ & 0,080 \\
\cline { 2 - 4 } & Não & $11(44 \%)$ & $11(23,9 \%)$ & \\
\hline
\end{tabular}

Fonte: Dados do autor, 2019

Os resultados obtidos pela falta de controle da PA, apesar da utilização de terapia medicamentosa com anti-hipertensivos pode sugerir um tipo de PRM (Problemas Relacionados a Medicamentos), visto que a efetividade destes medicamentos se encontra deficiente, uma vez que os entrevistados disseram ter adesão ao medicamento. É necessário que haja, portanto, um acompanhamento de perto com estes voluntários para que possa ser investigado o real motivo da inefetividade destes medicamentos e assim buscar solucionar este problema individual.

Em um estudo realizado com 270 pacientes hipertensos, foi observado que houve a adesão de $63 \%$ dos participantes ao tratamento com anti-hipertensivos em pessoas com hipertensão e, quando o déficit de adesão, foi notado que causou-se, primordialmente, por descuidos na tomada da medicação, evidenciando um problema de cunho cultural (ALBUQUERQUE et al., 2018).

Em relação à utilização de medicamentos, 14,08\% $(n=10)$ dos que participaram desta pesquisa afirmaram possuir algum tipo de RAM (reação adversa ao medicamento) como: náusea, vertigem, cefaléia, dor localizada no estômago, entre outros. Em contrapartida, foi verificado através das entrevistas que a adesão do uso de produtos naturais faz decair o uso de medicamentos quando necessário.

Segundo Barroso et al. (2021) para o controle da pressão arterial, o tratamento medicamentoso é uma opção válida, no entanto, se faz necessário também as mudanças dos hábitos alimentares e no estilo de vida em geral, e devem ser prioridade para o paciente sempre que possível. Para melhora no controle da HAS, podem ser utilizadas estratégias que consistem em intervenções organizacionais centradas no

RC: 106017

Disponível em: https://www.nucleodoconhecimento.com.br/saude/quilombola-do-curiau 
paciente e seus determinantes de saúde, sendo lideradas por equipes multidisciplinares, objetivando melhorar a qualidade do cuidado à Hipertensão e consequentemente a qualidade de vida (JARDIM et al., 2020).

Entre os voluntários, foi observado que 33,8\% (n=23) apresentaram aumento dos níveis pressóricos ( $\geq 140 / 90 \mathrm{mmHg})$ e dentre estes, 19,7\% ( $\mathrm{n}=14)$ tinham diagnóstico de hipertensão arterial. Dentre os participantes com pressão arterial alterada, 43,4\% $(n=10)$ eram mulheres e 56,5\% (n=13) eram homens. Houve diferença estatisticamente significativa $(p=0,009)$ entre os gêneros quanto a classificação das amostras em relação a elevação dos níveis pressóricos, havendo maior nível de PA elevada entre os homens, evidenciando alguns fatos notados na atenção à saúde dos homens, onde os indivíduos do sexo masculino demoram mais tempo para buscar assistência em saúde e, com isto, surge o diagnóstico tardio de diversas patologias, inclusive a HAS.

Neste sentido, Song et. al (2020) afirma que os homens têm níveis mais baixos de percepção da hipertensão e uma maior incidência desta doença em comparação com mulheres da mesma idade antes da sexta década de vida.

Quanto à classificação dos voluntários que possuem diagnóstico de HAS, não houve diferença significativa entre os gêneros $(p=0,813)$. É possível observar que neste estudo o sexo não apresenta um fator de risco para HAS na comunidade do Curiaú.

No estudo de Lobo et al. (2017) verificaram-se maiores prevalências de HAS nos indivíduos com menor escolaridade, independentemente dos anos estudados e do sexo, já na análise estratificada por sexo, verificou-se que nas mulheres de menor renda as prevalências de HAS foram maiores.

Em relação aos entrevistados que apresentaram elevação dos níveis pressóricos (com e sem diagnóstico de hipertensão), apenas 1,4\% $(n=1)$ com idade entre 18 a 39 anos apresentou elevação da PA. Entre 30 a 59 anos, 21,1\% $(n=15)$ obtiveram valores alterados de PA e em indivíduos com idade igual ou acima de 60 anos, 18,3\% (n=13) 
apresentaram valores acima dos considerados normais. Diante dos resultados observados houve associação significativa entre faixa etária e elevação dos níveis pressóricos neste estudo $(p=0,015)$ (Quadro 3). É importante ressaltar que 2 voluntários não tiveram interesse em aferir a PA no momento da pesquisa.

Quadro 3 - Dados de elevação de níveis pressóricos na população em estudo.

\begin{tabular}{|c|c|c|c|c|c|c|}
\hline \multirow{3}{*}{$\begin{array}{l}\text { Idade } \\
18-29\end{array}$} & \multicolumn{3}{|c|}{ HIPERTENSOS } & \multicolumn{3}{|c|}{ PA ELEVADA } \\
\hline & Sim & Não & P- Valor & Sim & Não & $\begin{array}{l}\text { P- } \\
\text { Valor }\end{array}$ \\
\hline & - & $\begin{array}{l}11 \\
(15,4 \%)\end{array}$ & 0,002 & $1(1,4 \%)$ & $10(14 \%)$ & 0,015 \\
\hline 30- 59 & $\begin{array}{l}11 \\
(15,4 \%)\end{array}$ & $\begin{array}{l}27 \\
(36,6 \%)\end{array}$ & & $\begin{array}{l}15 \\
(21,1 \%)\end{array}$ & $\begin{array}{l}22 \\
(30,9 \%)\end{array}$ & \\
\hline$\geq 60$ & $\begin{array}{l}13 \\
(19,3 \%)\end{array}$ & $9(12,6 \%)$ & & $\begin{array}{l}13 \\
(18,3 \%)\end{array}$ & $8(11,2 \%)$ & \\
\hline Total & $\begin{array}{l}24 \\
(33,8 \%)\end{array}$ & $\begin{array}{l}47 \\
(66,1 \%)\end{array}$ & & 29 (42\%) & $\begin{array}{l}40 \\
(57,9 \%)\end{array}$ & \\
\hline
\end{tabular}

Fonte: Dados do autor, 2019

Em relação à ingestão de bebida alcoólica e tabagismo, os voluntários responderam às perguntas com relação a frequência de sua utilização. Dentre os entrevistados: 32,3\% ( $n=23)$ fazem uso esporadicamente de bebida alcoólica, 8,4 \% ( $n=6)$ consomem álcool semanalmente e $3(n=4,2 \%)$ bebem diariamente. Por fim, 39 $(54,9 \%)$ dos entrevistados disseram não fazer uso de bebida alcoólica. Sobre o consumo de cigarro, apenas 1,4\% ( $n=1)$ dos voluntários fuma esporadicamente, $2,8 \%$ $(n=2)$ fazem uso semanalmente, $7 \%(n=5)$ afirmaram fumar diariamente e $88,7 \%$ $(n=63)$ dos participantes disseram não fazer uso de cigarro (Quadro 4). 
Quadro 4- Relação do consumo de bebida alcoólica e cigarro na população em estudo.

\begin{tabular}{|l|l|l|l|l|}
\hline VARIÁVEIS & HOMENS & \multicolumn{2}{l|}{ MULHERES } \\
\hline Álcool & $\mathrm{N}$ & $\%$ & $\mathrm{~N}$ & $\%$ \\
\hline Esporadicamente & 6 & 8,4 & 17 & 23,9 \\
\hline Semanalmente & 4 & 5,6 & 2 & 2,8 \\
\hline Diariamente & 2 & 2,8 & 1 & 1,4 \\
\hline Não faz uso & 13 & 18,3 & 26 & 36,6 \\
\hline Total & 25 & 35,2 & 46 & 64,7 \\
\hline Cigarro & $\mathrm{N}$ & $\%$ & $\mathrm{~N}$ & $\%$ \\
\hline Esporadicamente & 0 & 0 & 1 & 1,4 \\
\hline Semanalemente & 2 & 2,8 & 0 & 0 \\
\hline Diariamente & 2 & 2,8 & 3 & 4,2 \\
\hline Não faz uso & 21 & 29,5 & 42 & 59,1 \\
\hline Total & 25 & 35,2 & 46 & 64,7 \\
\hline
\end{tabular}

Fonte: Dados do autor, 2019.

Estima-se que o consumo excessivo de álcool seja responsável por cerca de 10-30\% dos casos de HAS e em torno de $6 \%$ da mortalidade de todas as causas no mundo (BARROSO et al., 2021.) Na análise de Santos et al. (2019), o tabagismo foi um dos fatores de risco que apresentaram associação com HA, havendo um percentual total de tabagistas de $37,18 \%$. Portanto, o tabagismo é reconhecido como um fator de risco para o surgimento da HA em adultos.

\section{CONSIDERAÇÕES FINAIS}

Estudo com grupos considerados vulneráveis são escassos, logo, destaca-se a importância deste trabalho para a comunidade acadêmica e sociedade em geral, pois teve como objetivo analisar as características epidemiológicas que possam estar associadas à HAS na população da Comunidade Quilombola do Curiaú. A identificação dos indivíduos hipertensos pode permitir o rastreamento e a RC: 106017

Disponível em: https://www.nucleodoconhecimento.com.br/saude/quilombola-do-curiau 
sensibilização de um grupo com o maior risco cardiovascular e de progressão para outras comorbidades ou piora do quadro da doença existente.

Dessa forma, levando em consideração que o Ministério da Saúde conceitua saúde como bem-estar biopsicossocial envolvendo vários aspectos, o estudo da Hipertensão Arterial Sistêmica em grupos vulneráveis faz-se pertinente. Dentre os vários parâmetros aqui abordados, destacou-se a escolaridade, idade e o sexo dos indivíduos. Portanto, respondendo à questão norteadora: Qual a prevalência e os fatores associados de HAS na Comunidade Quilombola do Curiaú? Dentre os resultados de prevalência de HAS encontrados neste estudo, foram considerados diversos fatores existentes, o que amplia a análise das prevalências entre diferentes populações e lugares. No entanto, este estudo revelou que a HAS é um problema de saúde importante na comunidade do Curiaú e, em destaque aos indivíduos do sexo feminino, pois foram os que mais apresentaram a doença. Também foi detectado maior prevalência de hipertensão em indivíduos com idade $\geq 60$ anos, sendo uma faixa etária considerada como fator de risco para a HAS devido ao processo de envelhecimento.

Concomitante a isso, ao analisar os voluntários que apresentaram elevação dos níveis pressóricos, é possível observar que indivíduos do sexo masculino apresentaram elevação em relação a idade. Pessoas na faixa etária entre 30 a 59 anos foram os que mais apresentaram a PA elevada. Isto deve ser levado em consideração, pois apresentam maior risco de desenvolver HAS, devendo ser monitorados frequentemente.

Os resultados do presente estudo, quando comparados com outros trabalhos em remanescentes de afrodescendente, demonstram a prevalência de HAS na Comunidade Quilombola do Curiaú, associada a fatores de risco conhecidos e específicos, voltando a atenção para a necessidade de implementação de políticas públicas que abranjam a necessidade de investigação dos determinantes de saúde e suas implicações em relação a assistência em saúde aos quilombolas, além de 
programas que considerem a adesão à terapia medicamentosa, com foco na melhoria da qualidade de vida e promoção de saúde pública da população desta comunidade.

\section{REFERÊNCIAS}

ALBUQUERQUE, Nila Larisse Silva de et al. Associação entre acompanhamento em serviços de saúde e adesão terapêutica anti-hipertensiva. Revista Brasileira de Enfermagem, v. 71, p. 3006-3012, 2018.

ANDRADE, Silvânia Suely de Araújo et al. Prevalência de hipertensão arterial autorreferida na população brasileira: análise da Pesquisa Nacional de Saúde, 2013. Epidemiologia e Serviços de Saúde, v. 24, p. 297-304, 2015.

BARROS, Marilisa Berti; CÉSAR, Chester Luiz; CARANDINA, Luana; TORRE, Graciella

Dalla. Desigualdades sociais na prevalência de doenças crônicas no Brasil, PNAD2003. Ciênc. saúde coletiva, 11(4):911-926, 2006.

BARROSO, Weimar Kunz Sebba et al. Diretrizes Brasileiras de Hipertensão Arterial. 2020. Arquivos Brasileiros de Cardiologia, v. 116, p. 516-658, 2021.

BRANDÃO, Andréa A. et al. Conceituação, epidemiologia e prevenção primária. Brazilian Journal of Nephrology, v. 32, p. 1-4, 2010.

JARDIM, Thiago Veiga et al. Controle da Pressão Arterial e Fatores Associados em um Serviço Multidisciplinar de Tratamento da Hipertensão. Arquivos Brasileiros de Cardiologia, v. 115, p. 174-181, 2020.

KURIAN, Anita; CARDARELLI, Kathryn. Racial and ethnic diferences in cardiovascular disease risk factors: a systematic review. Ethn Dis., 17(1):143-152, 2007. 
LINDHORST, Jane; ALEXANDER, Nichole; BLIGNAUT, Juliet; RAYNER, Brian. Differences in hypertension between blacks and whites: an overview. Cardiovasc $\mathbf{J}$ Afr., 2007;18(4):241-247, 2007.

LOBO, Larissa Aline Carneiro et al. Tendência temporal da prevalência de hipertensão arterial sistêmica no Brasil. Cadernos de Saúde Pública, v. 33, 2017.

MENEZES, Thiago de Castro; PORTES, Leslie Andrews; SILVA, Natália Cristina de Oliveira Vargas. Prevalência, tratamento e controle da hipertensão arterial com método diferenciado de busca ativa. Cadernos Saúde Coletiva, v. 28, p. 325-333, 2020.

MONTEIRO, Ariane Alice Fernandes et al. Estudo sobre a adesão ao tratamento de hipertensão arterial sistêmica na UBSF de Três Poços. Brazilian Journal of Health Review, v. 3, n. 1, p. 1289-1305, 2020.

PRATES, Elton Junio Sady et al. Características clínicas de clientes com hipertensão arterial e diabetes mellitus. Rev. enferm. UFPE on line, p. [1-10], 2020.

SANTOS, Angélica Viana Rocha et al. Perfil sociodemográfico do adulto quilombola com distúrbios hiperglicêmicos. Brazilian Journal of Health Review, v. 3, n. 6, p. 19007-19018, 2020.

SANTOS, Deyse Mirelle; PRADO, Beatriz Santana; OLIVEIRA, Cristiane Costa; ALMEIDA-SANTOS, Marcos Antonio. Prevalence of Systemic Arterial Hypertension in Quilombola Communities, State of Sergipe, Brazil. Arq. Bras. Cardiol., 113(3):383390, 2019.

SANTIAGO, Emerson Rogério Costa et al. Prevalence of systemic arterial hypertension and associated factors among adults from the semi-arid region of Pernambuco, Brazil. Arquivos brasileiros de cardiologia, v. 113, p. 687-695, 2019. 
SONG, Juan-Juan; MA, Zheng; WANG, Juan; CHEN, Lin-Xi; ZHONG, Jiu-Chang. Gender Differences in Hypertension. J Cardiovasc TransI Res, 13(1):47-54, 2020.

Enviado: Janeiro, 2022.

Aprovado: Janeiro, 2022. 